\title{
Letter to the Editor on Endovascular Revascularization with Stent Implantation in Patients with Acute Mesenteric Ischemia due to Acute Arterial Thrombosis: Clinical Outcome and Predictive Factors
}

\author{
Augusto Lauro $^{1}$ (D) $\cdot$ Paolo Sapienza ${ }^{2}$
}

Received: 3 August 2021/Accepted: 5 August 2021

(C) Springer Science+Business Media, LLC, part of Springer Nature and the Cardiovascular and Interventional Radiological Society of Europe (CIRSE) 2021

\section{Dear Editor,}

We read with great interest the article of Pedersoli et al. [1] recently published in an issue of the journal. The authors retrospectively described their experience on the emergency treatment of acute mesenteric ischemia with endovascular stenting of the superior mesenteric artery (SMA) and/or celiac trunk (CA). A total of 17 of the 40 patients $(42.5 \%)$ presented with bowel necrosis $(2 / 17)$ or ischemia (15/17) on pre-interventional computed tomography. Two out of 40 patients (5\%) underwent surgery before stent deployment. Revascularization of the target vessel was successful in 36/40 patients (90\%). After stenting, 25 out of 40 patients $(62.5 \%)$ underwent abdominal laparoscopy or laparotomy; specifically, in 12 patients (48\%) the ischemic bowel was resected, and in the remaining 13 patients (52\%) no additional surgery was needed or possible. Thirty-day-overall-mortality rate was 62.5\%: 10 patients died because of septic shock and 15 because of cardiogenic shock. Although mortality of the present series was high, no predictors for outcome were clearly identified. Conversely, in our experience [2] on 63 patients affected by acute superior mesenteric artery occlusion (mainly due to thromboembolism associated with non-hemodynamically significant atherosclerotic plaque at the origin of the superior mesenteric artery) and

Augusto Lauro

augusto.lauro@uniroma1.it

1 Department of Surgical Sciences, "Sapienza" University Policlinico Umberto I, Viale Regina Elena 324, 00161 Rome, Italy

2 Department of Surgery "Pietro Valdoni", "Sapienza" University, Rome, Italy treated as an emergent open surgical approach, $32(50.8 \%)$ patients were alive at discharge from hospital; notwithstanding, the overall postoperative complication rate was $50.8 \%$ in an elderly population (mean age 80 years old). Since in our emergency surgery team are present surgeons with vascular capabilities, we preferentially performed a surgical thrombectomy or, if indicated, a bypass from the aorta, eventually associated with bowel resection, followed by a direct anastomosis or stoma creation. A second surgical look was required in $15.8 \%$ of the patients. It is well known that acute thrombosis of the Ostia of the CA or SMA is associated with the worst prognosis, with an inhospital mortality rates up to $87 \%$ [3] because of the irreversible damage to the bowel wall occurring soon after the beginning of symptoms [4]. Therefore, a prompt diagnosis and timely intervention are crucial to avoid bowel necrosis, larger intestinal resections, sepsis, and death. Furthermore, in our study bowel loop dilation at pre-interventional CT scan was highly predictive of bowel ischemia, and mean time interval between onset of symptoms and surgery was less than six hours (349 min). In conclusion, we think that the interactions between several different specialties (interventional radiologists, vascular, and abdominal surgeons) might be time-consuming; therefore, we strongly support [5] the establishment of an intestinal stroke center.

\section{Declarations}

Conflicts of interest The authors declare that they have no conflicts of interest. 


\section{References}

1. Pedersoli F, Schönau K, Schulze-Hagen M, et al. Endovascular Revascularization with Stent Implantation in Patients with Acute Mesenteric Ischemia due to Acute Arterial Thrombosis: Clinical Outcome and Predictive Factors. Cardiovasc Intervent Radiol. 2021;44:1030-8.

2. Lauro A, Sapienza P, Vaccari S, et al. The surgical management of acute bowel ischemia in octogenarian patients to avoid Short Bowel Syndrome: a multicenter study. G Chir. 2019;40:405-12.

3. Schoots IG, Koffeman GI, Legemate DA, et al. Systematic review of survival after acute mesenteric ischaemia according to disease aetiology. Br J Surg. 2004;91:17-27.
4. Tilsed JVT, Casamassima A, Kurihara H, et al. ESTES guidelines: acute mesenteric ischaemia. Eur J Trauma Emerg Surg. 2016;42:253-70.

5. Nuzzo A, Maggiori L, Ronot M, et al. Predictive Factors of Intestinal Necrosis in Acute Mesenteric Ischemia: Prospective Study from an Intestinal Stroke Center. Am J Gastroenterol. 2017;112:597-605.

Publisher's Note Springer Nature remains neutral with regard to jurisdictional claims in published maps and institutional affiliations. 